\title{
Diversidad y abundancia de primates y sus amenazas en el interfluvio de los ríos Napo y Putumayo, Amazonía peruana
}

\section{Diversity and abundance of primates and their threats in the interfluvium of the Napo and Putumayo rivers, Peruvian Amazonia}

\author{
Rolando Aquino ${ }^{1 *}$, Luis López ${ }^{2}$, Iris Arévalo ${ }^{3}$ y Jesús Daza ${ }^{2}$
}

\author{
1 Facultad de Ciencias Biológicas/Universidad Nacional Mayor de San Marcos, Lima, Perú \\ 2 Universidad Nacional de la Amazonía Peruana, Iquitos, Perú \\ 3 Universidad Científica del Perú, Iquitos, Perú \\ *Autor para correspondencia \\ Email Rolando Aquino: raquinoy2005@yahoo.es \\ Email Luis López: luislopezramirez24@qmail.com \\ Email Iris Arévalo: irisarevaloo@qmail.com \\ Email Jesús Daza: faris.lobito500@hotmail.com
}

\section{Resumen}

En el nororiente de la Amazonía peruana todavía existen áreas con escasa información sobre primates, siendo una de ellas el interfluvio entre los ríos Napo-Putumayo, lo que motivó la conducción de este estudio para determinar su diversidad y abundancia, así como identificar las amenazas para sus poblaciones. Para este propósito se realizaron censos por transectos en octubre del 2007 , setiembre del 2013 y noviembre del 2014 en tres sitios de muestreo. En $1040 \mathrm{~km}$ de transectos recorridos fueron observados 308 grupos pertenecientes a nueve especies, siendo Leontocebus nigricollis el más común (109 grupos) y Alouatta seniculus el más escaso (16 grupos). Grupos más pequeños de Lagothrix lagothricha lagothricha (8-11 individuos) y A. seniculus (3-5 individuos) fueron observados en Tamboryacu considerado como el sitio de muestreo de mayor perturbación. Asimismo, la densidad poblacional más baja estimada para $L$. I. lagothricha y $A$. seniculus también correspondió a este sitio de muestreo con 3.8 indiv. $/ \mathrm{km}^{2}$ y 1.6 indiv. $/ \mathrm{km}^{2}$, respectivamente, mientras que para el resto de especies no hubo mayores diferencias entre los sitios de muestreo. Entre las actividades, la caza y extracción de madera son las principales amenazas y responsables para la escasa población de A. seniculus y I. I. lagotricha, principalmente en la cuenca del río Napo.

Palabras clave: Alouatta seniculus; Lagothrix lagothricha lagothricha; Leontocebus nigricollis; caza; extracción de madera.

\section{Abstract}

Within the northeastern Peruvian Amazonia remain areas with scarce information on primates, one of them being the interfluvium between the Napo and Putumayo rivers. This lack of information motivated us to conduct a study to determine the diversity and abundance of primates within the area, as well as to identify the threats, which inhibit these primate species populations. For this purpose, we conducted transect censuses in three sampling sites in October 2007, September 2013 and November 2014. In 1040 km of transect walks we observed 308 groups of nine primate species, the most common being Leontocebus nigricollis (109 groups) and the rarest being Alouatta seniculus (16 groups). Smaller groups of Lagothrix lagothricha lagothricha (8-11 individuals) and $A$. seniculus (3-5 individuals) were observed in Tamboryacu, a majorly disturbed sampling site. Likewise, the lowest population densities estimated for $L$. I. lagothricha and $A$. seniculus corresponded to this same sampling site with 3.8 indiv. $/ \mathrm{km}^{2}$ and 1.6 indiv. $/ \mathrm{km}^{2}$, respectively, while for the remaining species there were no major differences among the sampling sites. Among the activities, hunting and logging are the predominant threats responsible for the scarce populations of $A$. seniculus and I. I. lagothricha, mainly in the Napo River Basin.

Keywords: Alouatta seniculus; Lagothrix lagothricha lagothricha; Leontocebus nigricollis; hunting; logging.

\section{Citación:}

Aquino R., L. López, I. Arévalo \& J. Daza. 2016. Diversidad y abundancia de primates y sus amenazas en el interfluvio de los ríos Napo y Putumayo, Amazonía peruana. Revista peruana de biología 23(3): 243 - 252 (Diciembre 2016). doi: http://dx.doi.org/10.15381/rpb.v23i3.12859

\section{Presentado: $\quad$ 19/08/2016}

Aceptado: $\quad 28 / 11 / 2016$

Publicado online: $20 / 12 / 2016$
Información sobre los autores:

RAY: planifico la investigacion. RAY, LLR, IAP, JMDH ejecutaron los registros de campo. RAY, IAP: análisaron los datos. RAY, LLR: redactaron el manuscrito. RAY, LLR, IAP, JMDH: aprobaron el manuscrito

Los autores no incurren en conflictos de intereses.

Fuentes de financiamiento:

El presente trabajo se realizó gracias al financiamiento The Mohamed bin Zayed Species Conservation Fund; Vicerrectorado de InvestigaciónUniversidad Nacional Mayor de San Marcos; Idea Wild.

Journal home page: http://revistasinvestigacion.unmsm.edu.pe/index.php/rpb/index

(C) Los autores. Este artículo es publicado por la Revista Peruana de Biología de la Facultad de Ciencias Biológicas, Universidad Nacional Mayor de San Marcos. Este es un artículo de acceso abierto, distribuido bajo los términos de la Licencia Creative Commons Atribución-NoComercial-Compartirlgual 4.0 Internacional.(http://creativecommons.org/licenses/by-nc-sa/4.0/), que permite el uso no comercial, distribución y reproducción en cualquier medio, siempre que la obra original sea debidamente citadas. Para uso comercial, por favor póngase en contacto con editor.revperubiol@gmail.com. 


\section{Introducción}

Los bosques amazónicos del nororiente peruano albergan una de las mayores riquezas de especies de la fauna y flora silvestre (Gentry 1988, Myers et al. 2000, Bass et al. 2010, Hice \& Velazco 2012), siendo los primates uno de los componentes más representativos de la fauna de mamiferos para esta parte de la Amazonía peruana, y que han sido objeto de diversos estudios para estimar su diversidad y abundancia, en particular en los ríos Curaray (Heymann et al. 2002, Aquino et al. 2005, 2014), Arabela (Kolowski \& Alonso 2012), Pucacuro (Aquino et al. 2000a), Alto Itaya (Aquino et al. 2009, 2012, OversluijsVásquez 2003) y bosques aledańos a la carretera Iquitos-Nauta (Aquino et al. 2013a). Sin embargo, aún existen áreas con vacíos de información como las cuencas de los ríos Pastaza, Morona y Santiago, por citar algunos; por lo que se desconoce la situación de los primates que habitan en esas áreas y en muchas otras del territorio amazónico peruano.

En cuanto al interfluvio de los ríos Napo-Putumayo, considerado como una de las eco-regiones de máxima prioridad para su conservación en el planeta (Olson \& Dinerstein 2002), los únicos estudios sobre diversidad y abundancia de primates fueron realizados en el río Ampiyacu (Freese et al. 1982), río Yuvineto en el límite con Colombia (Encarnación et al. 1990) y en el curso inferior del río Algodón (Aquino et al. 2007), el resto son inventarios rápidos en los ríos Ampiyacu, Apayacu y Yaguas (Montenegro \& Escobedo 2004), Yanayacu (afluente izquierdo del río Napo) y Algodón correspondiente al área de influencia de los Maijunas (Bravo 2010) y Gueppí próximo a la frontera con Colombia (Bravo \& Borman 2008).

El objetivo del presente estudio es determinar la diversidad y abundancia de primates que habitan en el interfluvio de los ríos Napo-Putumayo e identificar las amenazas para la supervivencia de sus poblaciones. Para el análisis e interpretación, los datos fueron obtenidos durante los censos conducidos en octubre del 2007 en el curso inferior del río Algodón, setiembre del 2013 en el curso superior del río Algodón y noviembre del 2014 en bosques aledaños al curso medio del río Tamboryacu. Adicionalmente se realizaron exploraciones de corta duración entre noviembre y diciembre del 2012 a los ríos Yanayacu y Santa María, afluentes izquierdos del río Napo y bosques aledańos a los centros poblados de Angoteros y Torres Causana, asentados en la margen izquierda del río Napo. El propósito de estas exploraciones fue constatar in situ el impacto de las actividades en las poblaciones de primates, en particular de la extracción de recursos forestales y caza.

Área de estudio.- $\mathrm{El}$ área corresponde al nororiente peruano y está delimitada por los ríos Napo, Amazonas y Putumayo (Fig. 1) y para fines de evaluación de los primates se definieron tres sitios de muestreo que estaban sometidos a diferentes niveles de perturbación; en la Tabla 1 se presenta información en detalle acerca de la ubicación geográfica, tipos de bosques predominantes, nivel de perturbación humana y distancia recorrida en cada censo $(\mathrm{km})$. Los sitios de muestreo fueron:

Bajo Algodón: caracterizado por su baja perturbación humana debido a la existencia de comunidades indígenas, quienes se encargan de proteger sus bosques de los madereros ilegales y cazadores profesionales. La caza es esporádica y realizada únicamente por las comunidades del entorno, por lo que la fauna silvestre que habita los diversos tipos de hábitats es todavía rica y abundante.

Alto Algodón: caracterizado por su alta perturbación humana debido a la frecuente caza y extracción de árboles maderables por gente foránea. Los mamíferos mayormente cazados son los denominados de tamaño grande como Lagothrix lagothricha lagothricha y Alouatta seniculus entre los primates y Tapirus terrestris, Tayassu pecari, Pecari tajacu y Mazama americana entre los ungulados.

Tamboryacu: corresponde al sitio de muestreo más impactado por actividades de caza y extracción de madera y otros recursos forestales (cosecha de frutos y extracción de hojas de palmeras, lianas, bejucos, entre otros), siendo común la presencia de cazadores y madereros ilegales, quienes incluso han deforestado amplios espacios a orillas del río Tamboryacu para la instalación de campamentos (Fig. 2). En este sitio de muestreo la fauna mamífera fue muy escasa, en particular de animales considerados de tamaño grande y mediano, como los atélidos (L. lagothricha lagothricha y A. seniculus), cébidos (Cebus yuracus) y ungulados (T. terrestris, $M$. americana, T. pecari, entre otros).

\section{Material y métodos}

Censos.- El método utilizado fue el de transecto lineal (Burnham et al. 1980). En los sitios de muestreo fueron abiertos entre 5 a 6 trochas desde 4 a $5 \mathrm{~km}$ de longitud. El número y longitud de las trochas dependió de los tipos de hábitats presentes (bosque alto, palmal de planicie, varillal, pantano arbóreo, entre otros) y de las condiciones del piso (tierra firme o inundado). Los censos fueron de ida y vuelta, desde las 06:15 a 12:00 h y desde las 14:00 a 17:30 (hora local). Tres grupos cada uno integrado por un investigador y un asistente de campo se movilizaron diariamente por los distintos transectos a una velocidad promedio de $1 \mathrm{~km} /$ hora, con paradas cada cierto trecho por dos a tres minutos con la finalidad de explorar los diferentes estratos y detectar cualquier

Tabla 1. Proporción (\%) y número $(\mathrm{N})$ de grupos de primates observados por sitios de muestreo en el interfluvio Napo-Putumayo.

\begin{tabular}{lccc}
\hline Sectores & Ríos Algodón-Putumayo & Ríos Napo-Algodón & Río Tamboryacu \\
\hline Sitios de muestreo & Bajo Algodón (1) & Alto Algodón (2) & Curso medio Tamboryacu (3) \\
Coordenadas geográficas (18 UTM) & $818792 / 9705328$ & $685661 / 9725768$ & $615776 / 9789950$ \\
Nivel de perturbación del bosque & Bajo & Alto & Muy alto
\end{tabular}

Tipos de bosques predominantes

Longitud recorrida $(\mathbf{k m})$
Terraza baja y media, incluye aguajales densos
Colina baja, terraza media y alta

421
Terraza alta y baja, incluye aguajales mixtos
210
409 


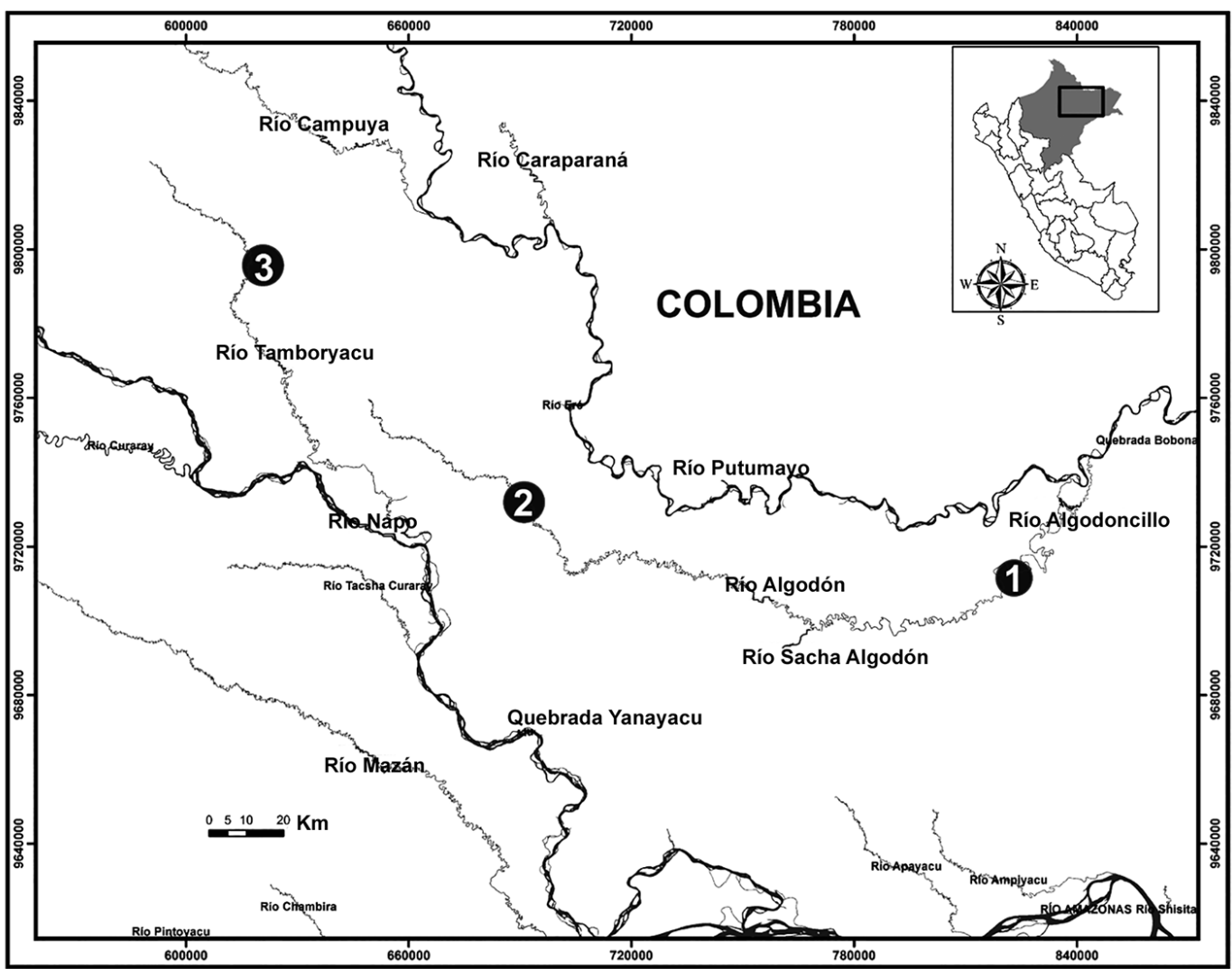

Figura 1. Ubicación de los sitios de muestreo en el interfluvio de los ríos Napo y Putumayo. Los números corresponden a la lista en la Tabla 1.

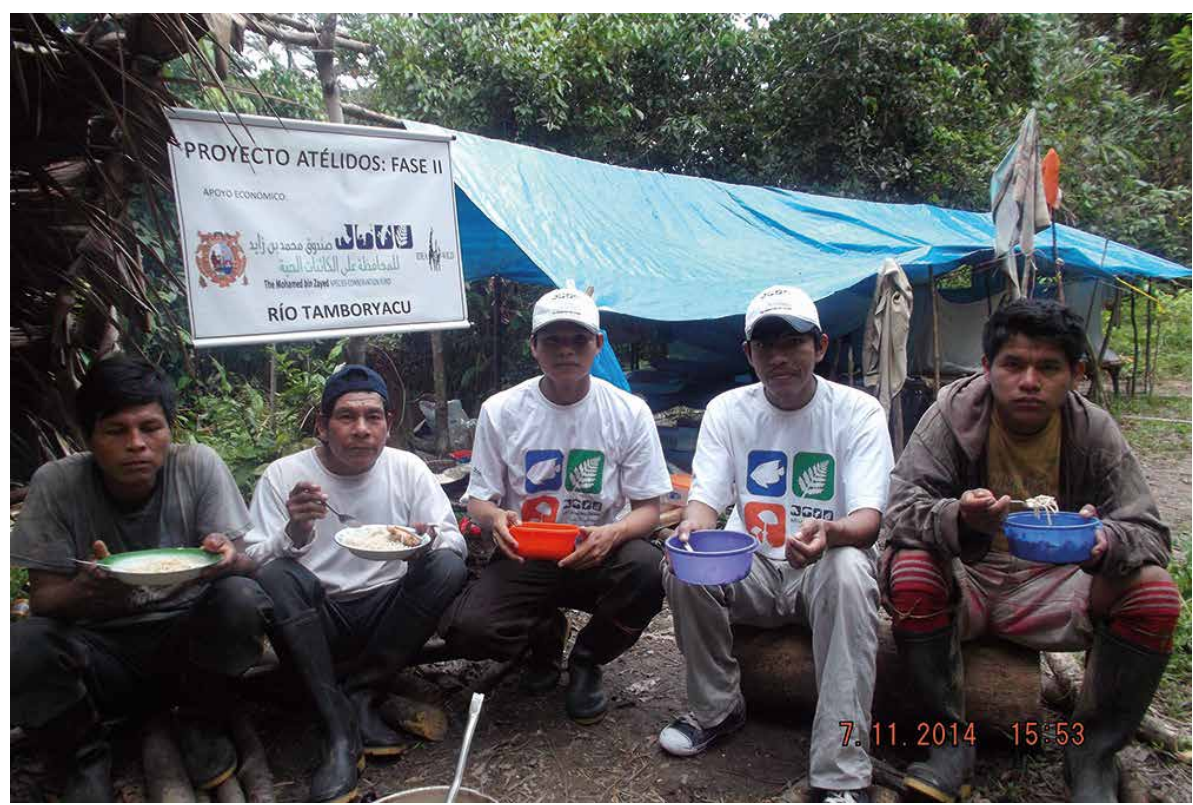

Figura 2. Carpas instaladas de los investigadores y personal de apoyo en espacios deforestados por madereros ilegales en el río Tamboryacu, noviembre, 2014.

movimiento y/o percibir los ruidos ocasionados por los saltos o caída de restos de frutos, ramas o bejucos secos que nos indicarían la presencia de primates. Cada vez que hubo contacto con un grupo procedimos a anotar en la libreta de campo los siguientes datos: especie, tamaño de grupo (cuando fue posible el conteo), presencia de infantes, distancia perpendicular del primer animal avistado al transecto, altura con respecto a los estratos del bosque, actividad al instante del contacto, tipo de hábitat y asociación con otras especies de primates.
Fueron excluidos de los censos Aotus vociferans y Cebuella pygmaea, la primera especie por su hábito nocturno y la segunda por su preferencia por el bosque de ribera. De un total de $1040 \mathrm{~km}$ recorridos, menor cobertura se hizo en el Bajo Algodón $(210 \mathrm{~km})$ debido a su lejanía con respecto a Iquitos y la dificultad para el acceso.

Identificación de amenazas.- Paralelo a los censos anotamos en la libreta de campo los campamentos y trochas en uso encon- 
Tabla 2. Proporción (\%) y número $(\mathrm{N})$ de grupos de primates observados por sitios de muestreo en el interfluvio Napo-Putumayo.

\begin{tabular}{|c|c|c|c|c|c|c|c|c|}
\hline \multirow{3}{*}{ Especies* } & \multicolumn{6}{|c|}{ Sitios de muestreo } & \multicolumn{2}{|c|}{ Total } \\
\hline & \multicolumn{2}{|c|}{ Bajo Algodón } & \multicolumn{2}{|c|}{ Alto Algodón } & \multicolumn{2}{|c|}{ Tamboryacu } & \multirow[b]{2}{*}{$\%$} & \multirow[b]{2}{*}{$\mathbf{N}$} \\
\hline & $\%$ & $\mathbf{N}$ & $\%$ & $\mathbf{N}$ & $\%$ & $\mathbf{N}$ & & \\
\hline Lagothrix lagothricha ${ }^{\dagger}$ & 16.4 & 14 & 21.2 & 21 & 4.0 & 5 & 13.0 & 40 \\
\hline Alouatta seniculus ${ }^{\dagger}$ & 6.0 & 5 & 6.1 & 6 & 4.0 & 5 & 5.2 & 16 \\
\hline Cebus yuracus ${ }^{\dagger}$ & 12.0 & 10 & 13.1 & 13 & 14.0 & 17 & 13.0 & 40 \\
\hline Pithecia hirsuta ${ }^{\dagger}$ & 14.0 & 12 & 13.1 & 13 & 15.3 & 19 & 14.3 & 44 \\
\hline Cheracebus lucifer ${ }^{\Phi}$ & 8.2 & 7 & 10.1 & 10 & 11.2 & 14 & 10.0 & 31 \\
\hline Saimiri macrodon ${ }^{\dagger}$ & 8.2 & 7 & 8.1 & 8 & 10.5 & 13 & 9.1 & 28 \\
\hline Leontocebus nigricollis ${ }^{\theta}$ & 35.2 & 30 & 28.3 & 28 & 41.0 & 51 & 35.4 & 109 \\
\hline Área total & 100 & 85 & 100 & 99 & 100 & 124 & 100 & 308 \\
\hline
\end{tabular}

* En orden decreciente a la masa corporal

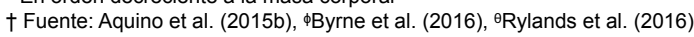

$\mathrm{N}$ : Total de grupos registrados

trados, casquillos de cartuchos en las trochas y campamentos, viales para el arrastre de madera redonda hacia el río, trochas carrozables, restos y despojos de animales cazados, frutos silvestres cosechados, encuentro con cazadores y madereros, entre otras observaciones de relevancia a las actividades de caza y tala. Adicionalmente se hicieron entre 2 a 3 entrevistas a los moradores más antiguos del caserío más cercano a los sitios de muestreo para indagar sobre otras actividades como exploraciones petroleras, construcción de carreteras, destino de los productos extraídos, en particular carne de monte y frutos silvestres, sitios de caza, entre otros. Asimismo, durante la visita a los caseríos tuvimos la oportunidad de constatar in situ la existencia de mascotas y otras evidencias de la caza como pieles y cráneos de primates.

Análisis de datos.- Con excepción de $A$. seniculus para los tres sitios de muestreo y L. l. lagothricha para el Tamboryacu, la densidad para el resto de especies fue analizada con el software DISTANCE versión 6.2 (Thomas et al. 2010). La densidad obtenida a través del Programa DISTANCE es confiable (Coeficiente de Variación menos del 30\%), excepto para $C$. yuracus y $S$. macrodon en Alto Algodón y Tamboryacu y C. lucifer en el Bajo Algodón y Alto Algodón (CV>30\%); no obstante, los datos presentan un ajuste adecuado al modelo establecido $(\mathrm{p}>0.05)$.

En cuanto a $A$. seniculus y $L$. l. lagothrica, los escasos registros para los sitios de muestreo antes mencionados (menores a siete muestras) no se ajustan al modelo para el uso del software DISTANCE, por lo que se ha recurrido al uso de la fórmula $\mathrm{D}=\mathrm{N} / 2 \mathrm{dL}$ (Burnham et al. 1980), donde $\mathrm{D}$ es la densidad (grupos y/o indiv. $/ \mathrm{km}^{2}$ ), $\mathrm{N}$ el número de grupos registrados a lo largo de los transectos, L la longitud total recorrida $(\mathrm{km})$ en los transectos y d la distancia perpendicular promedio del primer individuo avistado al transecto. El cálculo de indiv. $/ \mathrm{km}^{2}$ se obtuvo multiplicando D por el tamaño promedio del grupo, siendo excluidas las crías sin locomoción propia.

También fue calculado el número de avistamientos por 10 $\mathrm{km}$ de longitud recorrida. Para el análisis del tamaño promedio y rango de variación de grupos se han considerado únicamente a los grupos con conteo completo.

\section{Resultados}

Diversidad de especies.- En $1040 \mathrm{~km}$ de longitud recorrida fueron observados 308 grupos pertenecientes a 7 especies (Ta- bla 2). A ellos se suman $A$. vociferans y $C$. pygmaea observadas fuera de censos, con los cuales nuestro registro alcanza a nueve especies. Las mayores observaciones fueron para L. nigricollis con 109 grupos equivalente al 35,4\% del total, en tanto que A. seniculus fue la menos observada con solamente 16 grupos, que en términos porcentuales representó el 5.2\% (Tabla 2). En Tamboryacu, de los 124 grupos observados, 51 equivalente al $41 \%$ correspondió a $L$. nigricollis, mientras que $L$. l. lagothricha y $A$. seniculus fueron las menos observadas, ambas con 5 grupos.

Tamaño de grupo.- De acuerdo al nivel de perturbación de los bosques, el tamaño de grupo en atélidos y en algunos cébidos varió de más a menos; así, grupos más grandes de $L$. $l$. lagothricha (8 a 27 individuos) y $A$. seniculus (5 a 11 individuos) fueron observados en el Bajo Algodón considerado como el de menor impacto por actividades del hombre; lo contrario ocurrió en Tamboryacu, donde el tamaño de los grupos fluctuó entre 8 a 11 para la primera de las citadas y de 3 a 5 para la segunda especie (Tabla 3). El resultado fue similar para Cebus yuracus, en tanto que para las especies consideradas de tamaño pequeño como Cheracebus lucifer y L. nigricollis no hubo mayores diferencias.

Tasas de avistamiento y densidad poblacional.- En términos generales, ambas estimaciones fueron más altas para las especies en el Bajo Algodón y más bajas en Tamboryacu (Tabla 4). La densidad más alta en los tres sitios de muestreo fue para Saimiri macrodon y L. nigricollis y la más baja para C. lucifer en el Bajo Algodón y Alto Algodón con 1.5 indiv. $/ \mathrm{km}^{2}, A$. seniculus en Tamboryacu y Alto Algodón con 1.6 indiv. $/ \mathrm{km}^{2}$ y 3.5 indiv. $/ \mathrm{km}^{2}$ y L. l. lagothricha y $P$. hirsuta en Tamboryacu con 3.8 indiv. $/ \mathrm{km}^{2}$ y 1.8 indiv. $/ \mathrm{km}^{2}$, respectivamente (Tabla 4), resultados que indican que los primates que habitan en Alto Algodón y en particular en Tamboryacu están expuestos a una alta presión de caza.

Amenazas.- En el área de estudio, los primates y la fauna silvestre en general están expuestos a las amenazas que provienen de las siguientes actividades:

1. Caza: es la principal amenaza para los primates y otros componentes de la fauna mayor, siendo el Tamboryacu y Alto Algodón los más afectados por esta actividad, sitios donde con frecuencia encontramos brigadas de hasta 13 personas ejerciendo la caza, cuyos ruidos ocasionados por 
Tabla 3. Tamaño y rango de variación de grupos registrados durante los censos por sitios de muestreo en el interfluvio NapoPutumayo.

\begin{tabular}{|c|c|c|c|c|c|c|c|}
\hline \multirow[b]{2}{*}{ Sitios de muestreo } & \multirow[b]{2}{*}{$\begin{array}{l}\text { Lagothrix } \\
\text { lagothricha }\end{array}$} & \multirow[b]{2}{*}{$\begin{array}{l}\text { Alouatta } \\
\text { seniculus }\end{array}$} & \multicolumn{3}{|c|}{ Especies* } & \multirow[b]{2}{*}{$\begin{array}{c}\text { Saimiri } \\
\text { macrodon }\end{array}$} & \multirow[b]{2}{*}{$\begin{array}{c}\text { Leontocebus } \\
\text { nigricollis }\end{array}$} \\
\hline & & & $\begin{array}{c}\text { Cebus } \\
\text { yuracus }\end{array}$ & $\begin{array}{l}\text { Pithecia } \\
\text { hirsuta }\end{array}$ & $\begin{array}{c}\text { Cheracebus } \\
\text { lucifer }\end{array}$ & & \\
\hline \multicolumn{8}{|l|}{ Bajo Algodón } \\
\hline Tamaño promedio & 22.6 & 8.0 & 19.5 & 4.1 & 3.4 & $43.5^{\theta}$ & 5.6 \\
\hline D.S. & \pm 4.5 & \pm 2.6 & \pm 5 & \pm 0.7 & \pm 1.1 & & \pm 1.6 \\
\hline $\mathrm{N}$ & 3 & 4 & 2 & 7 & 5 & & 11 \\
\hline Rango & $8-27$ & $5-11$ & $16-23$ & $3-5$ & $2-5$ & $>31$ & $4-9$ \\
\hline \multicolumn{8}{|l|}{ Alto Algodón } \\
\hline Tamaño promedio & 19.0 & 5.0 & 12.5 & 4.0 & 3.4 & $43.5^{\ominus}$ & 5.9 \\
\hline D.S. & \pm 4.3 & \pm 2 & \pm 3.4 & \pm 1.4 & 1.6 & & \pm 1.8 \\
\hline $\mathrm{N}$ & 4 & 3 & 4 & 9 & 7 & & 10 \\
\hline Rango & $13-23$ & $3-7$ & $9-17$ & $2-6$ & $2-6$ & $>22$ & $4-10$ \\
\hline \multicolumn{8}{|l|}{ Tamboryacu } \\
\hline Tamaño promedio & 9.5 & 4.0 & $15.2^{\theta}$ & 3.3 & 2.6 & $43.5^{\ominus}$ & 5.7 \\
\hline D.S. & \pm 2.1 & \pm 1.4 & & \pm 1.2 & \pm 0.8 & & \pm 1.0 \\
\hline $\mathrm{N}$ & 2 & 2 & & 6 & 7 & & 12 \\
\hline Rango & $8-11$ & $3-5$ & $>14$ & $2-5$ & $2-4$ & $>49$ & $4-7$ \\
\hline
\end{tabular}

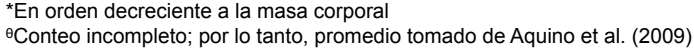

D.S: desviación estándar, N: Grupos con conteo completo.

los disparos eran escuchados incluso muy cerca de nuestros campamentos. En estos sitios, además de cazadores (Fig. 3), fue común encontrar campamentos con restos y despojos de animales, casquillos vacíos, residuos de frutos silvestres y hasta fardos de "carne de monte". Este mismo patrón fue observado en las exploraciones realizadas por los ríos Yanayacu, Santa María, cabeceras del río Algodón y otros afluentes del río Napo.
2. Extracción de madera: considerada como la segunda amenaza de importancia para la sobrevivencia de los primates y otros componentes de la fauna mayor. La extracción ilegal de madera es más frecuente en Tamboryacu (Fig. 4), por lo que el bosque se encuentra sumamente perturbado debido a la existencia de numerosas trochas y viales, así como de campamentos amplios para albergar a más de 15 personas.

Tabla 4. Tasa de avistamientos y densidad poblacional de primates por sitios de muestreo.

\begin{tabular}{|c|c|c|c|c|c|c|c|}
\hline Sitios de muestreo & $\begin{array}{l}\text { Lagothrix } \\
\text { lagothricha }\end{array}$ & $\begin{array}{l}\text { Alouatta } \\
\text { seniculus }\end{array}$ & $\begin{array}{c}\text { Cebus } \\
\text { yuracus }\end{array}$ & $\begin{array}{l}\text { Pithecia } \\
\text { hirsuta }\end{array}$ & $\begin{array}{c}\text { Cheracebus } \\
\text { lucifer }\end{array}$ & $\begin{array}{l}\text { Saimiri } \\
\text { macrodon }\end{array}$ & $\begin{array}{l}\text { Leontocebus } \\
\text { nigricollis }\end{array}$ \\
\hline \multicolumn{8}{|l|}{ Bajo Algodón: } \\
\hline \# de grupos/10 km & 0.66 & 0.24 & 0.47 & 0.57 & 0.33 & 0.33 & 1.4 \\
\hline Individuos $/ \mathrm{km}^{2}$ & 18.6 & 7.4 & 14.2 & 4.2 & 1.5 & 37.7 & 22.5 \\
\hline$\% \mathrm{CV}$ & 13 & & 11 & 17 & 30 & 29 & 18 \\
\hline Chi-cuadrado & 0.1 & & 0.2 & 0.4 & 0.2 & 0.7 & 0.4 \\
\hline \multicolumn{8}{|l|}{ Alto Algodón: } \\
\hline \# de encuentros/10 km & 0.5 & 0.14 & 0.31 & 0.31 & 0.24 & 0.19 & 0.66 \\
\hline Individuos $/ \mathrm{km}^{2}$ & 16.3 & 3.5 & 6.7 & 5.0 & 1.5 & 19.8 & 15.8 \\
\hline$\% \mathrm{CV}$ & 25.9 & & 30.2 & 22.0 & 35.0 & 40.6 & 29.3 \\
\hline Chi-cuadrado & 0.9 & & 0.3 & 0.4 & 0.5 & 0.7 & 0.6 \\
\hline \multicolumn{8}{|l|}{ Tamboryacu: } \\
\hline \# de encuentros $/ 10 \mathrm{~km}$ & 0.12 & 0.12 & 0.41 & 0.46 & 0.34 & 0.32 & 1.2 \\
\hline Individuos $/ \mathrm{km}^{2}$ & 3.8 & 1.6 & 8.6 & 1.8 & 1.9 & 34.1 & 18.6 \\
\hline$\% \mathrm{CV}$ & & & 32.0 & 19.3 & 27.6 & 32.5 & 13.4 \\
\hline Chi-cuadrado & & & 0.5 & 0.3 & 0.2 & 0.5 & 0.4 \\
\hline
\end{tabular}

*En orden decreciente a la masa corporal 


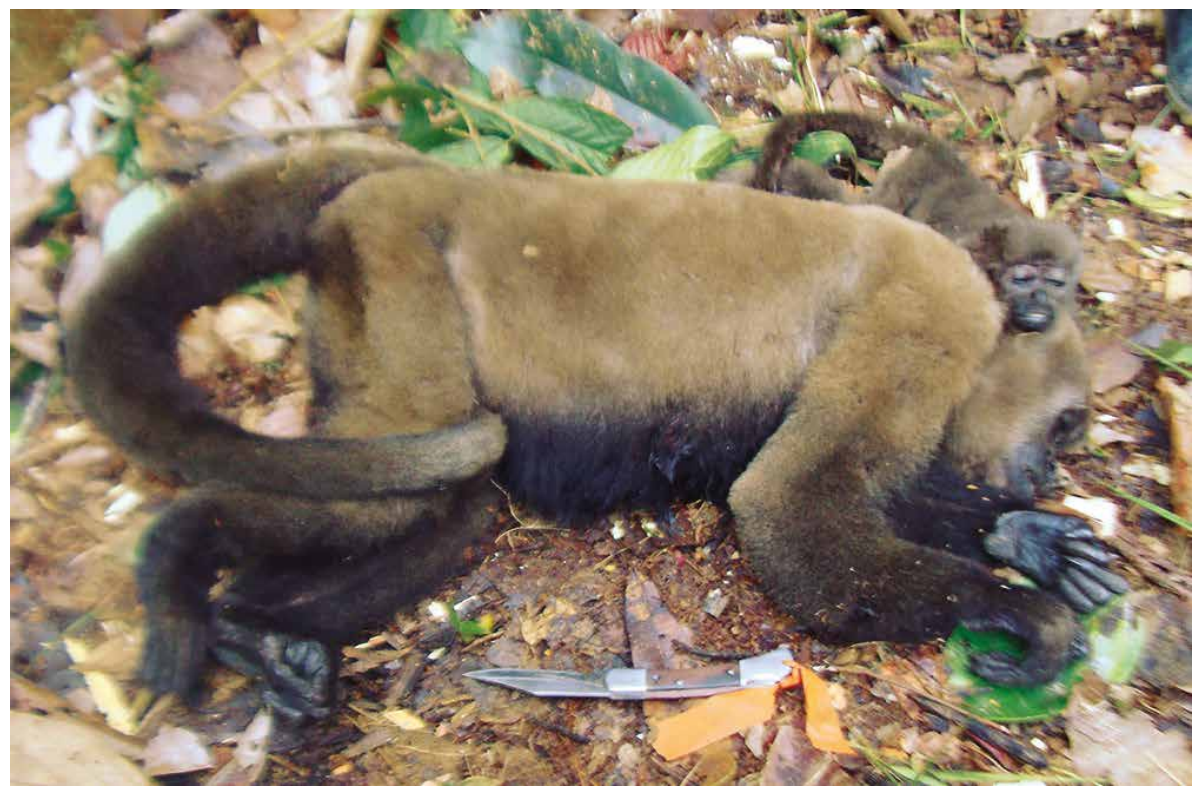

Figura 3. Ejemplar adulto de L. I. lagothricha cazado en el río Alto Algodón para consumo de subsistencia, setiembre 2013.

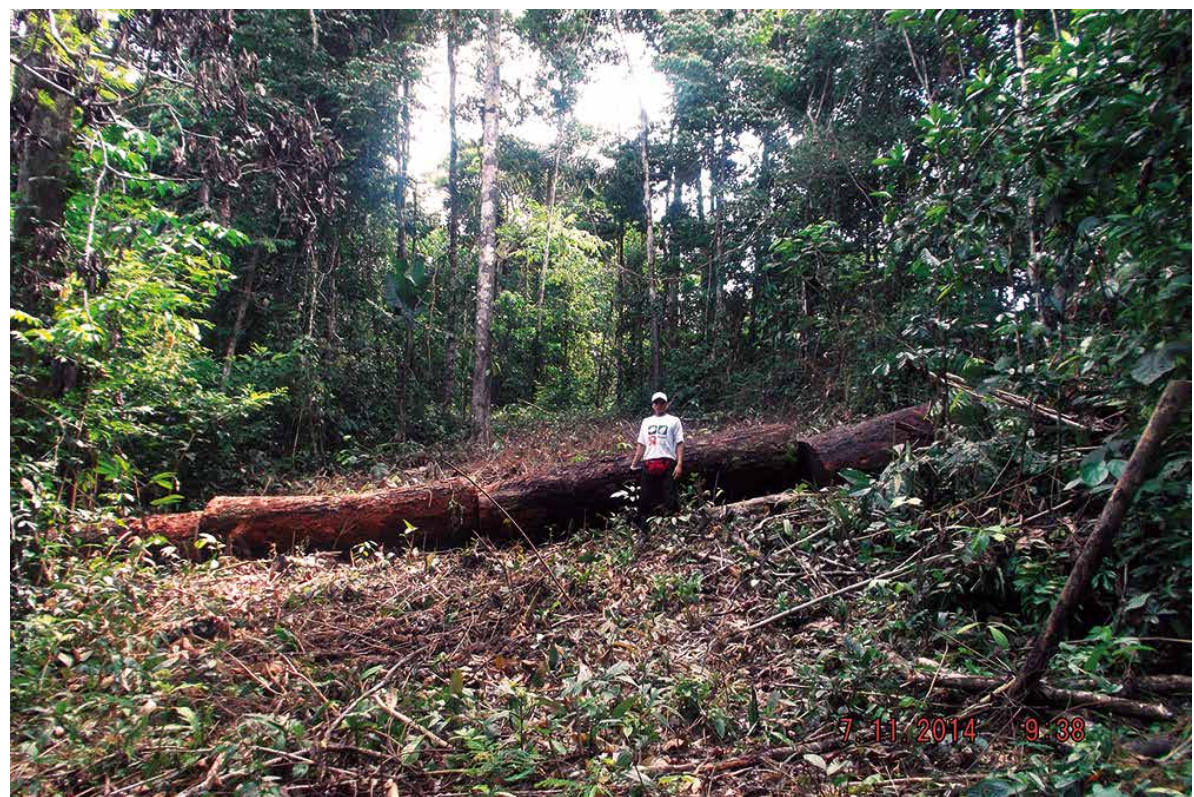

Figura 4. Deforestación para la extracción ilegal de madera en el río Tamboryacu, noviembre, 2014.

3. Cosecha de frutos silvestres: es otra de las amenazas a tener en cuenta. Entre los frutos silvestres extraídos, en particular del Alto Algodón y Tamboryacu figuran principalmente Mauritia flexuosa "aguaje" (Arecaceae), Oenocarpus bataua "ungurahui" (Arecaceae) y Couma macrocarpa "leche huayo" (Apocynaceae). Estos frutos son los más apreciados en las ciudades para el consumo en sus diversas modalidades (como frutas, helados, refrescos y otros), pero coincidentemente son también los recursos alimenticios más importantes para los primates y ungulados, tanto en el área de estudio como en otras partes de la Amazonía baja. Los "dueños" de estos productos manifestaron que la mayor parte de la cosecha es para la venta en los mercados de Iquitos y una pequeña parte para el consumo de la familia.

\section{Discusión}

La diversidad de primates registrada en este estudio resulta inferior al número de especies que habitan en los ríos Pucacuro (Aquino et al. 2000a), Yanayacu (Bravo 2010), Curaray (Aquino et al. 2014), interfluvio de los ríos Tigre y Nanay (Aquino et al. 2015a) y Tamshiyacu-Tahuayo (Puertas \& Bodmer 1993). El número de especies coincide con las reportadas para el Parque Nacional Yasuní (Marsh 2004), Cuyabeno-Güeppi (Bravo \& Borman 2008) y Yaguas (Montenegro \& Escobedo 2004). Es más alto al número de especies registradas en el Ampiyacu (Freese et al. 1982) y Cuyabeno (Encarnación et al. 1990), todos localizados en el nor oriente amazónico. 
La lista de especies proporcionada en este estudio difiere en algunas con las reportadas por Montenegro \& Escobedo (2004) quienes consideran además de Saguinus nigricollis (ahora Leontocebus nigricollis) a Saguinus fuscicollis (ahora Leontocebus fuscicollis) para el río Yaguas (afluente del río Putumayo), con Bravo (2010) quien cita a Cebus apella (ahora Sapajus macrocephalus) para el río Yanayacu (afluente izquierdo del río Napo) y con Bravo y Borman (2008) quienes reportan a Callicebus discolor (ahora Plecturocebus discolor) para Redondococha, sector de Gueppí; especies que a nuestro criterio no son oriundas del área de estudio por no corresponder su área de distribución (Aquino \& Encarnación 1994, Tirira 2007, Aquino et al. 2015b), por lo que la presencia de estas especies se debería a "enclaves" originados por el cambio del curso de los ríos, tal como ocurrió con Aotus nancymaae al norte del río Marañón (Hershkovitz 1983, Aquino \& Encarnación 1988) y entre la quebrada Atacuari y el río Amazonas, próximo al trapecio Amazónico (Aquino obs. pers.) y $S$. boliviensis peruviensis entre los ríos Ucayali y Tapiche (Hershkovitz 1984, Silva et al. 1992), posibilidades que no fueron tomadas en cuenta por los autores que reportaron a $S$. macrocephalus, P. discolor y L. fuscicollis para el interfluvio de los ríos Napo y Putumayo. En el caso específico de L. fuscicollis citado para el río Yaguas, éste podría tratarse de L. fuscus, presente en el lado colombiano (Defler 2010) y que por cambio del curso del río Putumayo también podría encontrarse en bosques inundables del territorio peruano, por lo que sería de interés verificar su identidad.

Con excepción del Bajo Algodón, los rangos de tamaño de grupo observados para L. l. lagothricha, A. seniculus y C. yuracus en los otros sitios de muestreo son inferiores a los registrados en el río Curaray catalogado como de baja perturbación humana (Aquino et al. 2014), pero similares a los observados en áreas con alta presión de caza como el río Samiria (Soini 1986, 1990), cuenca media del río Tapiche (Bennett et al. 2001), interfluvio de los ríos Urumbamba y Tambo (Aquino et al. 2013b) y ríos Tigre y Nanay (Aquino et al. 2015a). En referencia al resto de especies, los rangos del tamaño de grupo son similares entre los sitios con mayor y menor presión de caza, lo que indica que estos primates considerados como de tamaño pequeño son muy poco cazados.

Sin considerar al Bajo Algodón, las densidades estimadas en los otros sitios de muestreo, en particular para L. l. lagothricha y $A$. seniculus son inferiores a los reportados para el río Curaray (Aquino et al. 2014a), quebrada Arabela (Kolowski \& Alonso 2012), bosques con moderada caza de la cuenca del río Samiria (Aquino et al. 2000b), Tapiche (Bennett et al. 2001) e interfluvio de los ríos Urubamba y Tambo (Aquino et al. 2013b). No obstante, las densidades aquí calculadas son mayores a las estimadas para el río Pucacuro (Aquino et al. 2000a), bosques aledańos a la carretera Iquitos-Nauta (Aquino et al. 2013a) e interfluvio de los ríos Tigre y Nanay (Aquino et al. 2015a), que a diferencia del área de estudio están más impactadas por su proximidad a los centros poblados. Los resultados obtenidos en particular para los primates de tamaño pequeño también son mayores a los estimados para los bosques con caza intensiva del Parque Nacional Manú (Endo et al. 2010). Esta diferencia probablemente se debe a la poca cobertura por sitio de muestreo $(<250 \mathrm{~km}$ de longitud censada), aun cuando también podría tener relación con la alta presión de caza de los primates en general, considerando que las comunidades indígenas del sur oriente, entre ellos los machiguengas, tradicionalmente tienen preferencia por los primates como "carne de monte" (Aquino et al. 2013b). En cuanto a $C$. lucifer, la baja densidad estimada para el Bajo Algodón y Alto Algodón podría estar relacionado con el hábitat. De acuerdo con Aquino et al. (2013c), este primate habita preferentemente en comunidades vegetales de tipo "varillal", presente en el área de estudio, pero en pequeñas extensiones o "parches", aspecto que no fue tomado en cuenta durante los censos.

En el área de estudio, la caza y deforestación para extracción de madera y otros recursos forestales son las principales amenazas para los primates, en particular para los denominados de tamaño grande y mediano, coincidiendo así con los reportados para la cuenca del Alto Purús (Shoobridge 2003), Bajo Urubamba (Figueroa 2004), cabeceras de los ríos Ampiyacu, Apayacu, Yaguas y cuenca media del Putumayo (Montenegro \& Escobedo 2004), Reserva Comunal Matsés (Amanzo 2006) y Parque Nacional Güepí-Sekime (Bravo \& Borman 2008).

La caza es con fines comerciales y para susbsistencia. La primera está orientada principalmente a la obtención de ingreso económico, por lo que la "carne de monte" y los animales vivos (infantes) tienen como destino la ciudad de Iquitos. La caza de subsistencia es para el consumo de la familia, siendo utilizados mayormente animales de tamaño pequeño, entre ellos Dasyprocta fuliginosa entre los roedores, Dasypus spp. entre los cingulados y $C$. yuracus, $C$. lucifer y $P$. hirsuta entre los primates. La caza es muy intensa en casi la mitad del área existente en el interfluvio de los ríos Napo y Putumayo, lo que estaría originando una drástica disminución de las poblaciones de $L$. l. lagothricha y A. seniculus (ambos destinados a la venta), que incluso podría derivar en la extinción local de estas especies, tal como viene ocurriendo con Ateles belzebuth (Aquino et al. 2013d, 2015a) en el nor oriente peruano. La escasa presencia de estas dos especies en el Tamboryacu habría influido en el crecimiento poblacional de L. nigricollis, aun cuando esta especie fue también la más común en los otros sitios de muestreo. Afortunadamente muy cerca de los sectores con mayor presión de caza se crearon áreas de conservación como el Parque Nacional Gueppí-Sekime y las Reservas Comunales Humeki y Airo Pai, lo que garantizará la supervivencia de $L$. l. lagothricha y A. seniculus que son los más cazados entre los primates conjuntamente con los ungulados.

Sin embargo, es oportuno mencionar que la creación de áreas de conservación podrá garantizar la supervivencia siempre y cuando exista un control eficiente que impida o por lo menos reduzca el ingreso de cazadores furtivos. Otra forma de contrarrestar los impactos de la caza sería con la implementación de programas alternativos, entre ellos el de manejo sostenible con participación comunitaria tal como se viene llevando a cabo en el Área de Conservación Regional Tamshiyacu-Tahuayo (Bodmer 1993) y Reserva Nacional Pacaya Samiria (Bodmer et al. 1997). Sobre el particular, en el Área de Conservación Regional Ampiyacu-Apayacu se vienen aplicando herramientas metodológicas de monitoreo de fauna con el propósito de establecer líneas bases para la elaboración de planes de manejo de fauna bajo un sistema de cogestión (Puertas et al. 2016).

En referencia a los recursos maderables, además de la extracción ilegal en Tamboryacu, Alto Algodón y otros como 
Yanayacu y Santa María, también existen concesiones forestales ubicadas al sureste del Área de Conservación Regional Ampiyacu-Apayacu, próximas a la frontera con Colombia (OSINFOR 2013), de donde se extraen madera de manera legal e ilegal, sin tomar en cuenta que muchas de estas especies maderables cumplen un rol importante en la dieta de los primates, particularmente de los atélidos, tal como se demostró en una concesión forestal en la Amazonía boliviana donde Ateles chamek se alimentaba con frecuencia de los frutos de tres especies de árboles maderables, formando parte importante de su dieta alimenticia (Felton et al. 2010).

Por otro lado, la alteración y pérdida de bosques ya sea por extracción de recursos forestales o de hidrocarburos traen como consecuencia la disminución de poblaciones de primates, pérdida o fragmentación de hábitats, extinción local de especies, reducción en la frecuencia de dispersión de semillas y escasa población de árboles alimenticios (Peres 1997, Nuñez-Iturri \& Howe 2007, Stevenson \& Aldana 2008, Aquino et al. 2015a). Más allá de estas consecuencias, está la inminente desaparición o pérdida de primates y ungulados como dispersores en el largo plazo. Sin embargo, Mayor et al. (2015) sostienen que las poblaciones de primates y otros mamíferos en la cuenca media y alta del río Yavarí-Mirí no declinaron ante las actividades de extracción de madera, quienes además hacen referencia que estas poblaciones pueden recuperarse debido a la baja presión de caza y que estos resultados podrían extrapolarse en otras concesiones forestales. Al respecto, esta proposición se debería de tomar con cierta cautela, poque va depender mucho de la forma en que se realiza la extracción de madera, la misma que puede variar mucho entre áreas, es decir puede ser muy selectiva o puede ser mucho más amplia.

Frutos de M. flexuosa "aguaje", O. bataua "ungurahui" y C. macrocarpa "leche huayo" tienen una alta demanda en los mercados de Iquitos. Estos recursos alimenticios son también importantes para los primates y ungulados, tanto en el área de estudio como en otras partes de la Amazonía peruana (Bodmer 1990, Aquino \& Encarnación 1999, Aquino 2005, Bowler \& Bodmer 2011, Charpentier et al. 2015). La extracción de frutos no solamente ocasiona escasés de recursos alimenticios para los animales sino también incremento de la deforestación y consecuente alteración de los hábitats porque generalmente se obtienen los frutos derribando el árbol.

Finalmente, en el área de estudio una de las potenciales amenazas para la fauna mayor, en particular para los componentes de los atélidos y ungulados será la construcción de la carretera de aproximadamente $95 \mathrm{~km}$ de longitud que conectará a las cuencas de los ríos Napo y Putumayo. El asentamiento de caseríos previsto hasta $5 \mathrm{~km}$ en ambos lados del eje de la carretera traerá como consecuencia mayor presión de caza y deforestación.

\section{Agradecimiento}

Agradecemos a The Mohamed bin Zayed Species Conservation Fund por el apoyo económico para la conducción del estudio orientado al inventario y evaluación de Atélidos y de otros primates en el nororiente de la Amazonía peruana. A la Universidad Nacional Mayor de San Marcos (UNMSM) por su contribución económica para la adquisición de materiales de campo. Nuestro reconocimiento a Idea Wild por la donación de algunos materiales que fueron de mucha utilidad en el campo. Especial deferencia a los guías de campo de las localidades de Puerto Arica y Nuevo Libertador asentados en el río Napo y Esperanza en el río Putumayo, con quienes compartimos gratas experiencias durante las actividades de campo.

\section{Literatura citada}

Amanzo J. 2006. Mamíferos medianos y grandes. En: C. Vriesendorp, N. Pitman, J.I. Rojas Moscoso, B. A. Pawlak, C.L. Rivera Chavez, L. C. Mendes, C.M. Vela y R. P. Fasabi,eds. Perú: Matsés. Rapid Biological Inventories Report 16. The Field Museum, Chicago. Pp. 98-106.

Aquino R. \& F. Encarnación. 1988. Population densities and geographic distribution of night monkeys (Aotus nancymae and Aotus vociferans) (Cebidae: Primates) in northeastern Peru. American Journal of Primatology 14: 375-381. http:// dx.doi.org/10.1002/ajp.1350140407

Aquino R. \& F. Encarnación. 1994. Primates of Peru/ Los Primates del Perú. Primate Report 40: 1-130.

Aquino, R. \& F. Encarnación. 1999. Observaciones preliminares sobre la dieta de Cacajao calvus ucayalii en el Nor-Oriente peruano. Neotropical Primates 7(1): 1 - 5 .

Aquino R., R.E. Bodmer \& E. Pezo. 2000a. Evaluación de primates en la cuenca alta del río Pucacuro, Amazonía Peruana. En: J. Espinoza, F. San Martín y E. Montoya, eds. La Primatología en el Perú. Vol. 2. Master Graf. Editores, Lima. Pp. 92-100.

Aquino R., R.E. Bodmer \& G. Gil. 2000b. Impacto de la caza en poblaciones de primates de la cuenca del río Samiria, Reserva Nacional Pacaya Samiria. En: F. San Martín Howard y M. García Podestá, eds. La Primatología en el Perú. Vol. 2. Master Graf. Editores, Lima. Pp. 81-91.

Aquino, R. 2005. Alimentación de mamíferos de caza en los aguajales de la Reserva Nacional Pacaya Samiria (Iquitos, Perú). Revista Peruana de Biología 12 (3): 417 - 425. http:// dx.doi.org/10.15381/rpb.v12i3.2416

Aquino R., C. Ique \& H. Gálvez. 2005. Reconocimiento preliminar de la densidad y estructura poblacional de Saguinus tripartitus Milne-Eduards en la Amazonía peruana. Revista Peruana de Biología 12(3): 435-440. http://dx.doi. org/10.15381/rpb.v12i3.2418

Aquino R., T. Pacheco \& M. Vásquez. 2007. Evaluación y valorización económica de la fauna silvestre en el río Algodón, Amazonía peruana. Revista Peruana de Biología 14(2): 187-192. http://dx.doi.org/10.15381/rpb.v14i2.1730

Aquino R., W. Terrones, R. Navarro, et al. 2009. Caza y estado de conservación de primates en la cuenca del río Itaya, Loreto, Perú. Revista Peruana de Biología 15(2): 33-39. http:// dx.doi.org/10.15381/rpb.v15i2.1719

Aquino R., C. Tuesta \& E. Rengifo. 2012. Diversidad de mamíferos y sus preferencias por los tipos de hábitats en la cuenca del río Alto Itaya, Amazonía peruana. Revista Peruana de Biología 19(1): 35-42. http://dx.doi.org/10.15381/rpb.v19i1.785

Aquino R., E. Charpentier \& G. Mendoza. 2013a. Diversidad y abundancia de primates en bosques del área de influencia de la carretera Iquitos-Nauta, Loreto, Perú. En: R. Aquino, E. Pezo Lozano y D.E. Gil Macedo, eds. Mamíferos de la Cuenca del Río Alto Itaya, Amazonía Peruana. Editorial Academia Española, Saarbrücken. Pp. 95-118.

Aquino R., F.M. Cornejo \& E.W. Heymann. 2013b. Primate abundance and habitat preferences on the lower Urubamba and Tambo rivers, central-eastern Peruvian Amazonia. Primates 54: 377-383. http://dx.doi.org/10.1007/s10329013-0357-3

Aquino, R.; W. Terrones; F. Cornejo \& E. Pezo. 2013c. Aspectos ecológicos y estructura poblacional del tocón negro (Callicebus torquatus) (Primates: Pitheciidae). En: R. Aquino, E. Pezo Lozano y D.E. Gil Macedo, eds. Mamíferos de la Cuenca del Río Alto Itaya, Amazonía Peruana. Editorial Academia Española, Saarbrücken. Pp. 150-182.

Aquino R., F.M. Cornejo, E. Pezo, et al. 2013d. Distribution and abundance of white-fronted spider monkeys, Ateles belzebuth (Atelidae) and threats to their survival in Peruvian Amazonia. Folia Primatologica 84: 1-10. http://dx.doi. org/10.1159/000345549 
Aquino R., L. López, G. García, et al. 2014a. Diversity, abundance and habitats of the primates in the río Curaray basin, Peruvian Amazonia. Primate Conservation 28: 1-8. http:// dx.doi.org/10.1896/052.028.0103

Aquino R., L. López, G. García, et al. 2015a. Situación actual de primates en bosques de alta perturbación del nororiente de la Amazonía peruana. Ciencia Amazónica 5(1): 50-60. http://dx.doi.org/10.22386/ca.v5i1.90

Aquino R., F. Cornejo, L. Cortés, F. Encarnación et al. 2015b. Primates del Perú: Guía de identificación de bolsillo. Conservation International, Arlington, Virginia; USA.

Bass M.S., M. Finer, C.N. Jenkins, et al. 2010. Global conservation significance of Ecuador's Yasuní National Park. PLoS One 5(1): e8767. http://dx.doi.org/10.1371/journal. pone. 0008767

Bennett C.L., S. Leonard \& S. Carter. 2001. Abundance, diversity, and patterns of distribution of primates on the Tapiche River in Amazon Peru. American Journal of Primatology 54: 119-126. http://dx.doi.org/10.1002/ajp.1017

Bodmer R. 1990. Fruit patch size and frugivory in the lowland tapir (Tapirus terrestris). Journal Zoological London 222: 121-128. http://dx.doi.org/10.1111/j.1469-7998.1990. tb04034.x

Bodmer R. 1993. Manejo de fauna Silvestre con las comunidades locales: El caso de la Reserva Comunal TamshiyacuTahuayo. Manuscrito, caso de estudio 12b, Universidad de Florida. 20 pp.

Bowler M. \& R.E. Bodmer. 2011. Diet and food choice in Peruvian red uakaris (Cacajao calvus ucayalii): selective or opportunistic seed predation?. International Journal of Primatology 32:1109-1122. http://dx.doi.org/10.1007/ s10764-011-9527-6.

Bravo A. 2010. Mamíferos. En: M.P. Gilmore, C. Vriesendorp, W.S. Alverson, A. del Campo, R. von May, C. López Wong y A. Ríos Ocha, eds. Perú: Maijuna. Rapid Biological and Social inventories Report 22. The Field Museum, Chicago. Pp. 90-96.

Bravo A. \& R. Borman. 2008. Mamíferos. En: W.S. Alverson, C. Vriesendorp, A. del Campo, D.K. Moskovits, D.F. Stotz, M. García y L.A. Borbor, eds. Ecuador, Perú: CuyabenoGüeppí. Rapid Biological and Social inventories Report 20. The Field Museum, Chicago. Pp. 105-111.

Burnham K.P., D.L. Anderson \& J.L. Laake. 1980. Estimation of density from line transects sampling of biological populations. Wildlife Monographs 72: 1-202.

Byrne H., A.B. Rylands, J.C. Carneiro, et al. 2016. Phylogenetic relationships of the New World titi monkeys (Callicebus): first appraisal of taxonomy based on molecular evidence. Frontiers in Zoology 1-25. https://doi.org/10.1186/ s12983-016-0142-4

Charpentier E., G. García \& R. Aquino. 2015. Uso y competición por plantas alimenticias entre Pithecia aequatorialis (Primates: Pitheciidae) y otros animales en la Amazonía peruana. Revista Peruana de Biología 22 (1): 1-8. https:// doi.org/10.15381/rpb.v22i2.11356

Defler T.R. 2010. Historia natural de los primates colombianos. Editorial Universidad Nacional de Colombia. Bogotá, Colombia. 609 pp.

Encarnación F., N. Castro \& P. de Rham. 1990. Observaciones sobre primates no humanos en el Río Yuvineto (Río Putumayo), Loreto, Peru. In: DGFF/IVITA/OPS, ed. La Primatología en el Perú Investigaciones Primatológicas (1973-1985). Imprenta Propaceb, Lima. Pp.68-79.

Endo W., C.A. Peres, E. Salas, et al. 2010. Game vertebrate densities in hunted and nonhunted forest sites in Manu National Park, Peru. Biotropica 42: 251-261. https:// doi.org/10.1111/j.1744-7429.2009.00546.x

Felton A.M., A. Felton, W.J. Foley, et al. 2010. The role of timber tree species in the nutritional ecology of spider monkeys in a certified logging concession, Bolivia. Forest Ecology and Management 259(8): 1642-1649. https://doi. org/10.1016/j.foreco.2010.01.042

Figueroa J. 2004. Mamíferos. En: C. Vriesendorp, L. Rivera Chávez, D. Moskovits y J. Shopland, eds. Perú: Megantoni. Rapid Biological Inventories Report 15. The Field Museum, Chicago. Pp. 110-118.
Freese C.H, P.G. Heltne, R.N. Castro \& G. Whitesides.1982 Patterns and determinants of monkey densities in Peru and Bolivia, with notes on distributions. International Journal of Primatology 3:52-90. https://doi.org/10.1007/ BF02693490

Gentry A.H. 1988. Tree species richness of upper Amazonian forests. Proceeding of the National Academy of Sciences of the United States of America 85(1): 156-59. https:// doi.org/10.1073/pnas.85.1.156

Hershkovitz P. 1983. Two new species of night monkeys, genus Aotus (Cebidae, Platyrrhini): A preliminary report on Aotus taxonomy. American Journal of Primatology 4: 209-243. https://doi.org/10.1002/ajp.1350040302

Hershkovitz P. 1984. Taxonomy of squirrel monkeys genus Saimiri (Cebidae, Platyrrhini): A preliminary report with description of a hitherto unnamed form. American Journal of Primatology 7: 155-210. https://doi.org/10.1002/ ajp. 1350070212

Heymann E.W., F. Encarnación \& J. Canaquín. 2002. Primates of the río Curaray, northern Peruvian Amazon. International Journal of Primatology 23(1): 191-201. https://doi. org/10.1023/A:1013262210863

Hice C.L. \& P.M. Velazco. 2012. The non-volant mammals of the Reserva Nacional Allpahuayo-Mishana. Loreto, Peru. Special Publications of the Museum of Texas Tech University, Number 60. Pp. 135.

Kolowski J.M. \& A. Alonso. 2012. Primate abundance in an unhunted region of the northern Peruvian amazon and the influence of seismic oil exploration. International Journal of Primatology 33: 958-971. https://doi.org/10.1007/ s10764-012-9627-y

Marsh L.K. 2004. Primate species at the Tiputini Biodiversity Station, Ecuador. Neotropical Primates 12(2): 75-78.

Mayor P., P. Pérez-Peña, M. Bowler, et al. 2015. Effects of selective logging on large mammal populations in a remote indigenous territory in the northern Peruvian Amazon. Ecology and Society 20(4): 36. http://dx.doi.org/10.5751/ ES-08023-200436.

Montenegro O. \& M. Escobedo. 2004. Mamíferos. En: N. Pitman, R.C. Smith, C. Vriesendorp, D. Moscovits, R. Piana, G. Kneli y T. Wachter, eds. Perú: Ampiyacu, Apayacu, Yaguas, Medio Putumayo. Rapid Biological and Social inventories Report 12. The Field Museum, Chicago. Pp. 163-169.

Myers N., R.A. Mittermeier, C.G. Mittermeier, et al. 2000. Biodiversity hotspots for conservation priorities. Nature 403: 853-858. https://doi.org/10.1038/35002501

Nuñez-Iturri G. \& H. F. Howe. 2007. Bushmeat and the fate of trees with seeds dispersed by large primates in a lowland rain forest in western Amazonia. Biotropica 39(3): 348354. https://doi.org/10.1111/j.1744-7429.2007.00276.x

Olson D.M. \& E. Dinerstein. 2002. The Global 200: priority ecoregions for global conservation. Annals of the Missouri Botanical Garden 89: 199-224. https://doi. org/10.2307/3298564

OSINFOR. 2013. Evaluación de áreas deforestadas y humedales en los departamentos de Loreto, Ucayali y Madre de Dios al año 2011. 1er edición. Solvima Graf SAC. Lima. 74 pp.

Oversluijs-Vásquez M.R. 2003. Animales de caza en la Zona Reservada Allpahuayo Mishana. Folia Amazónica 14(1): 7-16.

Peres C.A. 1997. Primate community structure at twenty western Amazonian flooded and unflooded forests. Journal of Tropical Ecology 13: 381-405. https://doi.org/10.1017/ S0266467400010580

Puertas P.E. \& R. Bodmer. 1993. Conservation of a high diversity primate assemblage. Biodiversity and Conservation 2:586-593. https://doi.org/10.1007/BF00051959

Puertas P.E., A. Pinedo, S. Soplín, et al. 2016. Evaluación poblacional de fauna silvestre orientada a los animales de caza en el Alto Yaguas, Área de Conservación Regional Ampiyacu Apayacu. Reporte interno IBC. Iquitos. 43 pp.

Rylands A.B., E.W. Heymann, A.J. Lynch, et al. 2016. Taxonomic review of the New World tamarins (Primates: Callitrichidae). Zoological Journal of the Linnean Society 1-26. https://doi.org/10.1111/zoj.12386 
Shoobridge D. 2003. Amenazas a la conservación socio ambiental de la región Alto Purús. En: M.R.P. Leite Pitman, N. Pitman y P. Álvarez, eds. Alto Purús: Biodiversidad, conservación y manejo. Center for Tropical Conservation. Lima. Pp. 165-176.

Silva B.T.F., M.I.C. Sampaio, H. Schneider, et al. 1992. Natural hybridization between Saimiri taxa in the peruvian Amazonia. Primates 33: 107-113. https://doi.org/10.1007/ BF02382766

Soini P. 1986. A synecological study of a primate community in the Pacaya-Samiria National Reserve, Peru. Primate Conservation 7: 63-71.

Soini P. 1990. Ecología y dinámica poblacional de pichico común Saguinus fuscicollis (Callitrichidae, Primates). In: DGFF/ IVITA/OPS, ed. La Primatología en el Perú Investigaciones Primatológicas (1973-1985). Imprenta Propaceb, Lima. Pp. 202-253
Stevenson P.R. \& A.M. Aldana. 2008. Potential effects of Ateline extinction and forest fragmentation on plant diversity and composition in the western Orinoco basin, Colombia. International Journal of Primatology 29: 365-377. https://doi.org/10.1007/s10764-007-9177-x

Tirira D.G. 2007. Mamíferos del Ecuador. Guía de campo. Ediciones Murciélago Blanco. Publicación Especial de los Mamíferos del Ecuador 6. Quito. 576 pp.

Thomas L., S.T. Buckland, E.A. Rexstad, J.L. Laake, et al. 2010. Distance software: design and analysis of distance sampling surveys for estimating population size. Journal of Applied Ecology 47: 5-14. https://doi.org/10.1111/j.13652664.2009.01737.x 